\title{
Advanced Micro and Nano Manufacturing Technologies Used In Medical Domain
}

\author{
M.S Packianather ${ }^{1}$, C.H. Le ${ }^{2}$, Dinh Trung Pham ${ }^{3}$, Hoai Quoc Le ${ }^{4}$ \\ ${ }^{1}$ School of Engineering, Cardiff University, Cardiff, United Kingdom \\ ${ }^{2}$ Faculty of Engineering and Science, University of Greenwich, Kent, United Kingdom \\ ${ }^{3}$ University of Phan Thiet, Phan Thiet, Vietnam \\ ${ }^{4}$ Saigon Hi-Tech Park - SHTP, Ho Chi Minh, Vietnam
}

\begin{abstract}
This paper focuses on the aspects of advanced manufacturing technologies, namely micro and nano manufacturing (MNM) capabilities which are particularly relevant to medical domain. In recent years, the so called disruptive technologies have enabled engineers and clinicians to collaborate in solving complex problems which require advanced MNM capabilities to develop medical applications. As a result what was nearly impossible a few years ago, due to limitations in machining and manufacturability of micro and nano scale artefacts, are now made possible thanks to innovative manufacturing processes and technologies. The potential medical applications of the new MNM methods are immense and in this paper four potential uses, namely as medical devices, lab on chips, and brain implants are presented and discussed. These works were based on different projects undertaken by researchers at Cardiff University, UK. The manufacturing costs, though initially high, are expected to reduce over time as the technologies mature and become more widely available. Introducing these MNM technologies and disseminating these results to healthcare engineering, for a better quality of medical diagnosis and treatments with cost-effective solutions, will greatly benefit the majority of population who live in the developing countries in receiving appropriate and affordable medical care to achieve improvements in their quality of life.
\end{abstract}

Keywords - Micro and nano manufacturing, implants, medical instrumentation, Lab on a Chip.

\section{INTRODUCTION}

Micro and nano-manufacturing (MNM) has been playing an important role in today's product design and development, not only by the requirement of increasingly sophisticated devices, structures and functional units, but also by the trend of decreasing component sizes, material usages and energy consumption of products $[1,2]$; especially it has directly contributed to the development of sophisticated medical devices and tools for complex medical diagnostics and treatments, as well as recent rapid advancements in bio technologies and nano medicine $[2,4]$. The new capabilities of MNM enable the design, fabrication, integration and assembly of miniature scale components with embedded actuation and intelligence for monitoring, carrying out selected procedures, diagnosis and prevention of medical conditions. The technology is now delivering personalised body parts, inserts, scaffolds, and markers for targeting the delivery of a particular drug in the most effective way.
The MNM technologies that are suitable for medical applications include additive, subtractive, disruptive, and hybrid approach [5]. In practice, non-contact methods are desirable due to the demands on achieving micro and nano dimensionality with a need for sterilisation before use within the human body and other medical applications. As more and more technologies become available in manufacturing engineering, we are able to achieve remarkable results in developments of high-values added products, through innovation and research. The need to find reliable and cost effective solutions are continuing to grow in the field of medical domain. In this paper, key aspects of advanced MNM technologies are presented and discussed, with the focus on the applications of MNM for medical product development. The benefits of these MNM technologies are demonstrated by different case studies. The rest of the paper is organised as follows. Section II presents reviews about the core MNM technologies that are important and necessary for medical product development. Section III presents case studies about applications of MNM to develop products for medical diagnosis and treatments. Finally, a brief conclusion and discussions are presented in Section IV.

\section{AdVANCED MICRO AND NANO MANUFACTURING TECHNOLOGIES FOR MEDICAL PRODUCT DEVELOPMENT}

\section{A. Micro Injection Moulding}

Micro Injection Moulding (MIM) is essentially a replication process. The MIM process for thermoplastic materials such as PC involves initially heating polymer granules that are contained within a hopper. Then the melted content is fed into a machine barrel where it is rotated and heated to a specified temperature. In the following step, the molten material is injected into a heated mould tool and allowed to cool. Afterwards, the solid plastic component is ejected from the mould tool. A schematic photograph of the Battenfeld Microsystem 50 MIM machine and a diagrammatic representation of the injection unit are shown in Fig.1.

\section{B. Nano imprint lithography}

(a) Laser Ablation

This is primarily a non-contact machining process. It involves the removal of material by targeting the area by a high power laser for short duration of time so that the energy absorbed will cause the material to heat and eventually to evaporate. The efficiency of this process will depend on the 
optical properties of the material, laser wavelength and pulse duration which could vary from milliseconds to femtoseconds. The shorter the pulse duration the better the end results of the machining process. This process is capable of machining a range of materials including ceramics. The mechanism of the above process is shown in Fig.2.

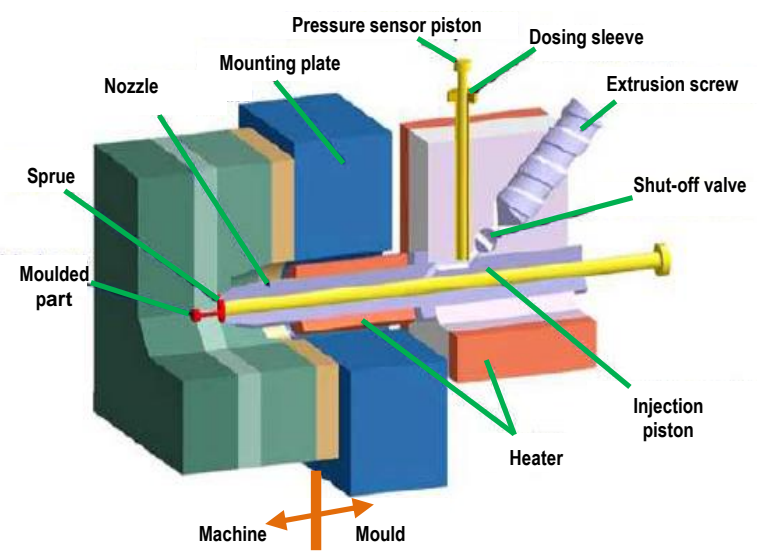

Fig. 1 Battenfeld Microsystem 50 showing different components of the process [10]

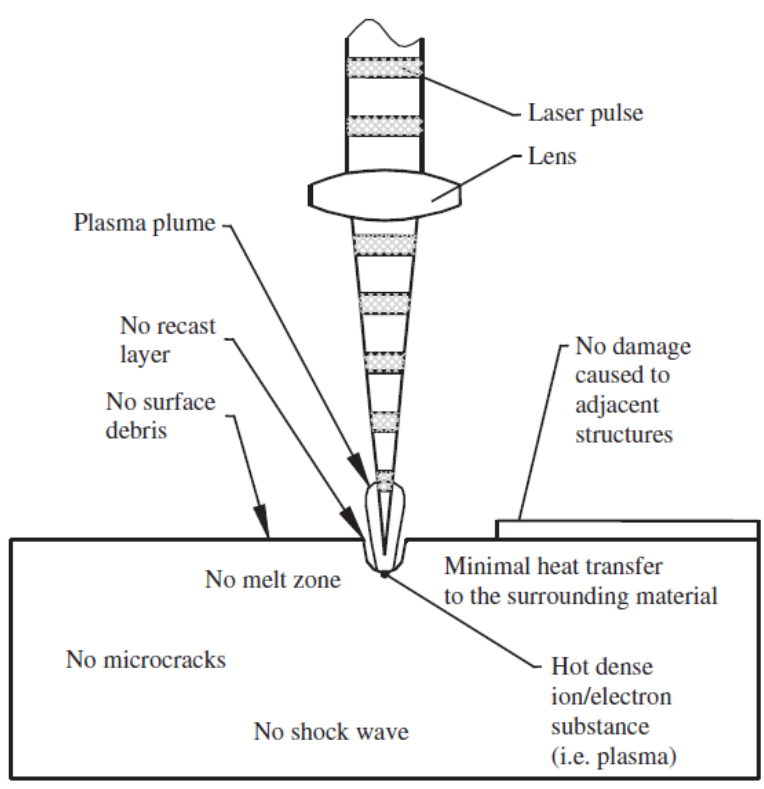

Fig. 2 Laser Ablation mechanism [8]

\section{(b) Nano Imprint Lithography}

This is primarily a thermal replication process similar to Hot Embossing where a heated mould is pressed against a polymer sheet, where heating and pressing are fundamental steps. In thermal Nano Imprint Lithography (NIL) nanoscale structures are moulded on thin spin-coated polymer films which are later used as masks in the replication process. It is a method of creating nanometer scale patters by mechanical deformation of imprint resist followed by curing by UV light as shown by the schematic process in Fig. 3 .

\section{(c) Micro Electro Discharge Machining:}

This is a structuring process capable of achieving micro and nanoscale features. Two electrodes namely the tool and the workpiece are immersed in a dielectric fluid and are conducted with current. As the electrodes get closer the dielectric breaks down and spark is formed which erodes the workpiece creating the desired pattern. The result of a repeated process of this discharge in a controlled manner is capable of achieving craters on the surface forming micro and nanoscale structures. The general principle of Electro Discharge Machining (EDM) process is shown in Figure 4.

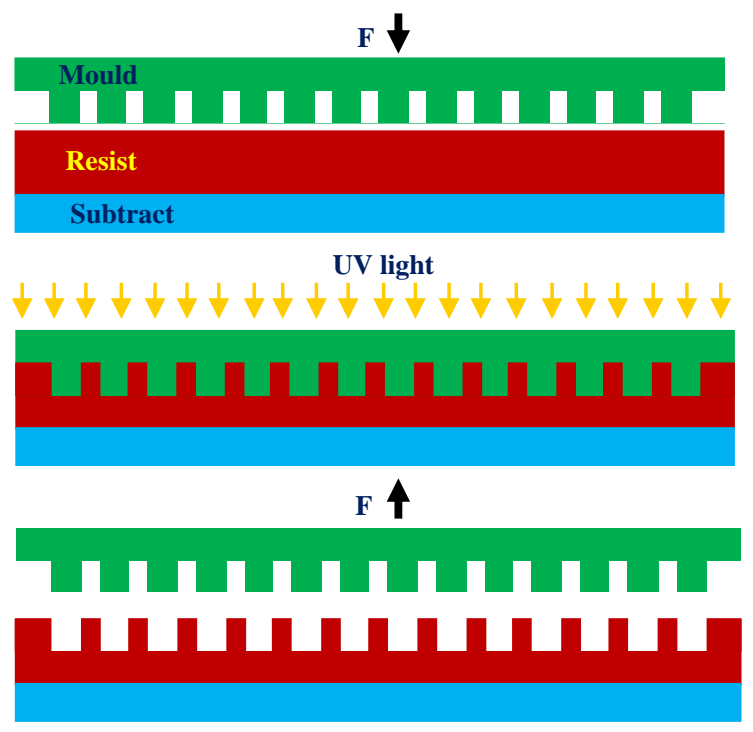

Fig. 3 Schematic diagram of UV light imprint lithography [7]

\section{CASe Studies ABOUT ADVANCED MiCRO AND NANO MANUFACTURING TECHNOLOGIES USED IN MEDICAL PRODUCT DEVELOPMENT}

\section{A. Micro needles}

Micro-needles are different to traditional hypodermic needles with the main advantage being concerned with the ability of a micro-needle to pierce the dermis of the skin in a non-invasive and painless way [11]. In addition to this, hypodermic needles should only be used by fully trained healthcare professionals, they must be disposed of safely, patient compliance may be considered to be an issue due to 'needle-phobia' and the risk of spread of blood-borne pathogens through needle re-use are cited as being significant 
issues [12]. In order to manufacture micro needles mould cavities were made in an array using laser ablation and were filled with molten polymer using the MIM machine. The simulation of the MIM process is shown in Fig. 5(a). The actual micro needle formed by MIM is shown in Fig. 5(b).

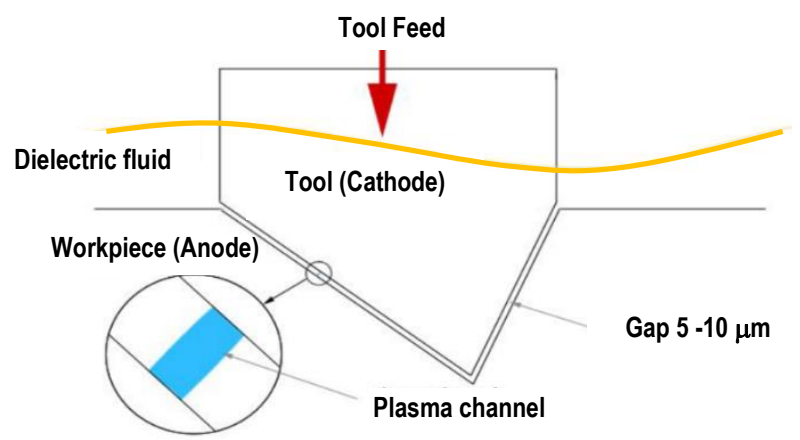

Fig.4 The general principle of electro discharge machining process [9]

\section{B. Multi-needle brain implant}

Researchers at Utah University have been developing a multiple-needle brain implant which is inserted into patient's brains in an area of the brain responsible for motor function. The dimension of these needles and their uniform array formation meant that it would be very challenging and near impossible to make it using the traditional machining techniques on a hard to machine material. Using micro EDM technique the implant the size of a match head containing 100 sensors were made using an extremely hard material namely tungsten carbide.

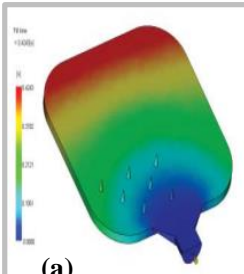

(a)
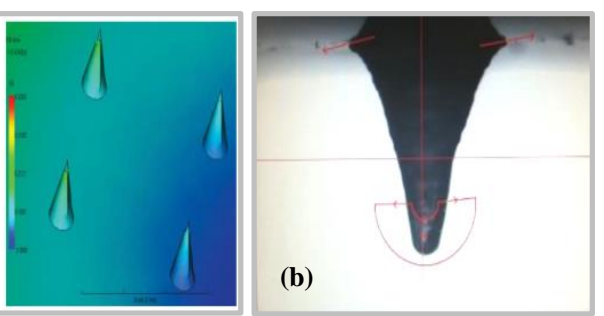

Fig. 5 (a): Simulation of the MIM process showing a mould with an array of o micro needles. (b): The micro needle formed by MIM process.

\section{Moth eye lens for medical diagnostics}

The need to understand night vision and capturing images in low light conditions have forced scientists to study the moth eye and how it utilises all the light available to fly around in the dark avoiding obstacles. To do this the moth eye is made up of an array of nanoscopic structures on the surface to prevent any light from reflecting off the surface. Using the conventional manufacturing methods it will be near impossible to build an artificial lens imitating the moth eye. However, using advanced manufacturing technologies such as focused ion beam and nano imprint lithography this has been made possible.

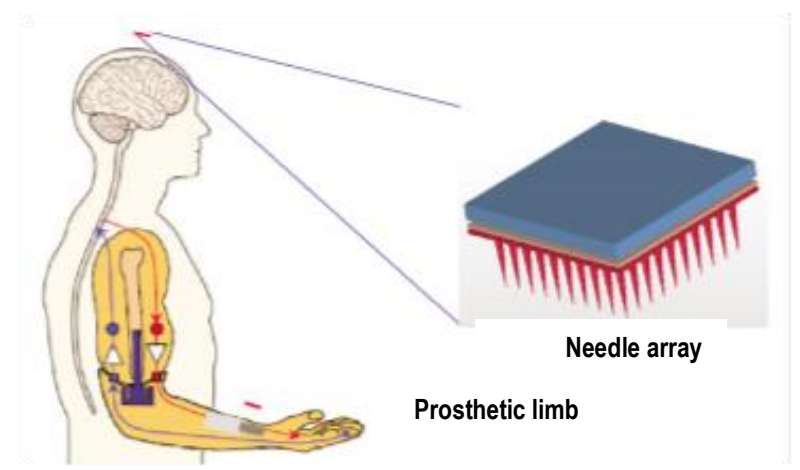

Fig. 6 An implant used as man/machine interface and prosthetic limb control system [13]

The individual lenses which are less than 100 nanometer in size were formed by nano-patterning using FIB (Fig. 7(a)) and later replicated using NIL (Fig. 8). These artificial moth eye lenses could be used optoelectronics and photovoltaic applications in semiconductors, including solar cells, where loss of light is a major problem. The lens also has potential uses in fibre optics, sensors and medical diagnostic devices.
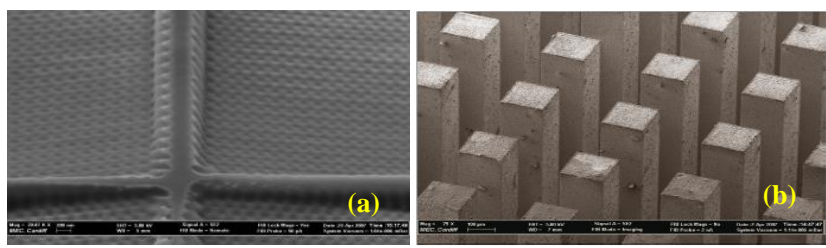

Fig. 7 (a): The microscopic image of the array of needles, each $120 \mathrm{mi}-$ crons width. (b) Individual nanoscopic lens structures formed using focused ion beam [14]

These sensors when inserted into the brain will be capable of receiving nerve signals to control prosthetic limbs. Figure 6 shows how the implant is inserted into an area of patient's brain responsible for motor action. The microscopic image of the multi-needle brain implant is shown in Fig. 7 (b).

\section{Lab on chips}

Often medical diagnostics takes a long time and requires bio samples to be transported from place to place. The new generation of diagnostic kit will provide an all-in-one lab on chip capability thanks to micro nano machining technologies. The system will be based on plasmonics, optics and micro-fluidics to create a fully integrated polymer based card type plasmonic enhanced SPR bio sensor. The micro fluidic channels and substrate layers were machined using technologies such as laser ablation to cater for the injection 
of the fluidic test sample into the sensor. The use of this lab on a chip sensor will increase sensitivity of the test, increase diagnostic capabilities while making it cost effective and suitable for disposing after one-off use. This can also be used for monitoring specific bio markers, allergy testing and hepatitis. Figure 9 shows the lab on a chip sensor concept.
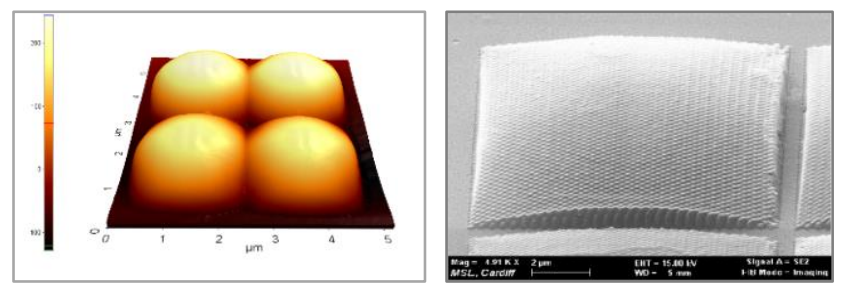

Fig.8 AFM image of a Step and Flash nano imprint lithography structure of the moth eye lens [14]

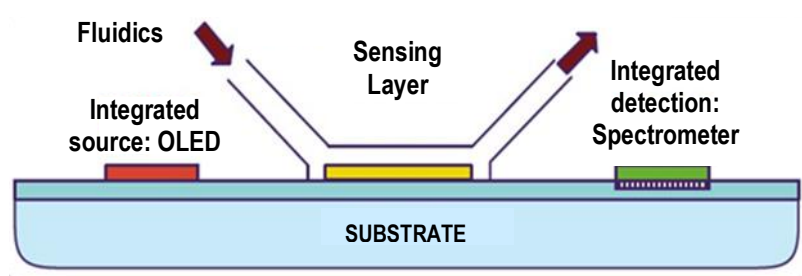

Fig.9 Lab on chip concept for medical diagnosis [15]

\section{DisSCUSION AND CONCLUSION}

In this paper, the key advanced MNM technologies are presented. The use of these technologies for development of medical applications is demonstrated with four case studies. These case studies have shown the benefits of engineering in medicine and how advanced MNM technologies could be used to improve medical care and make it more affordable. The technologies introduced could be used in providing similar solutions or to solve more challenging problems by an innovative approach.

Although there has been some steady progress in the development of MNM technologies there are still some challenges and obstacles which need to be addressed. These include the use of a range of materials and the creation of complex 3D features with high aspect ratios. Each technology offers certain advantages and disadvantages and could be broadly classified into scalable Additive Manufacturing, Direct Writing, and hybrid process. The cost and economy of scale is another issue which needs to be tackled to make the units personalised and replicable. Further, the sustainable design and manufacture of medical products with minimum material and energy usage for achieving zero waste and carbon footprint remains a real challenge.

Between 2005-2015, some 7.6 million people died from cancer, accounting for $13 \%$ of global deaths; and it is anticipated that 84 million people will die of cancer; and approx- imately $72 \%$ of cancer deaths occurred in low and middle income countries [3]. In addition, in low-income countries, the survival rates of cancers and related diseases are also much lower, largely because of delays in diagnosis leading to presentation with advanced disease. With the introduced MNM technologies, advanced lab-on-chips, biosensors and medical devices can be developed for a better quality of medical diagnosis and treatments with cost-effective solutions. This will greatly benefit the majority of population who live in the developing countries in receiving appropriate and affordable medical care to achieve improvements in their quality of life.

\section{ACKNOWLEDGMENT}

British Council - Newton Fund is acknowledged for their support.

\section{REFERENCES}

1. Lin Li et al. (2011) Laser nano-manufacturing - State of the art and challenges. CIRP Annals - Manufacturing Technology; 60(2): 735755.

2. Patil M et al. (2008) Future impact of nanotechnology on medicine and dentistry.Journal of Indian Society of Periodontlogy;12(2):34-40.

3. Cancer in Developing Countries: www.inctr.org [Access: April 2016]

4. Pimpin A. and Srituravanich W. (2012) Review on Micro- and Nanolithography Techniques and their Applications. Engineering Journal; 16(1): 37-55.

5. Vaezi M et al. (2013) The International Journal of Advanced Manufacturing Technology; 67(5): 1721-1754.

6. Lacan F. et al (2011) Manufacturing of an Array of Microneedles in a Steel Insert for Micro-Injection Moulding Using Laser Milling. Proceedings of the 8th International Conference on Multi-Material Micro Manufacture (4M 2011), 2011, pp336-339.

7. Omar F. (2013) Hot Embossing Process Parameters: Simulation and Experimental Studies. PhD Thesis, School of Engineering, Cardiff University, UK.

8. Petkov P.V. et al. (2007) Laser milling: pulse duration effects on surface integrity. Proc. IMechE Part B: J. Engineering Manufacture; 222: $35-45$.

9. Surleraux A. B. (2015) Numerical simulation and optimisation of micro-EDM using geometrical methods and machine learning. $\mathrm{PhD}$ Thesis, School of Engineering, Cardiff University, UK.

10. Tosello G. (2008) Precision moulding of polymer micro components". PhD Thesis, DTU department of Mechanical Engineering, Denmark.

11. Van der Maaden K et al. (2012) Microneedle technologies for (trans)dermal drug and vaccine delivery. Journal of Control Release; 161(2):645-55

12. Yeu-Chun Kim et al. (2012) Microneedles for drug and vaccine delivery. Advanced Drug Delivery Reviews; 64(14): 1547-1568.

13. Sensor could help amputees move limbs using their brain, Assistive Technologies (2009): 64: 6.

14. Brousseau E. B. et al. (2010) Some recent advances in multi-material micro- and nano- manufacturing. Int $\mathrm{J}$ Adv Manuf Technology; 47:161-180

15. Brousseau E. B. (2007) Recent Advances in micro manufacturing. ftp://ftp.cordis.europa.eu/pub/ims/docs/5-7-brousseau.pdf [Access: April 2016]

Author: Michael Packianather

Institute: Cardiff University

Street: The Parade

City: Cardiff

Country: UK

Email: PackianatherMS@ cardiff.ac.uk 